\title{
Propagation of VLF Waves Under Disturbed Conditions
}

\author{
B. Burgess
}

Ministry of Aviation, Royal Aircraft Establishment, Radio Department, S. Farnborough, Hampshire, England

\section{Introduction}

The very stable phase properties of VLF wave propagation over great distances are being currently considered or utilized for worldwide frequency comparisons, time synchronization, and long-range radio navigational aids. All these uses rely on the very stable properties of VLF propagation, and hence a knowledge of disturbed propagation conditions, their cause, and understanding is of practical importance.

Since the earliest days of longwave research, the effects on propagation of magnetic, solar, and other atmospheric disturbances have been known, though it is only in recent years that advances have been made in understanding them. The effect of a sudden ionospheric disturbance (SID) is well known for VLF propagation over short distances [Bracewell et al., 1951], and has been studied recently for long propagation paths [Chilton et al., 1962]. It will be shown in the present paper how use of this effect can be used to study the modes of propagation over ranges of 1,000 to $3,000 \mathrm{~km}$.

The effects of great magnetic storms had been observed as early as 1938 [Budden et al., 1939] and it had been noted that disturbed conditions had lasted for many days after the magnetic storm had ceased. Some recent measurements of GBR at Rome (path-length of $1,500 \mathrm{~km}$ ) under conditions of high magnetic activity, shown anomalous propagation conditions and are possibly indicative of effects associated with polar cap absorption events spreading as far south as $48^{\circ} \mathrm{N}$ (geomagnetic).

The effects of recent high altitude nuclear explosions, which are similar in many ways to those of magnetic storms activities are also briefly indicated.

\section{Observations of Disturbances}

\subsection{Sudden Ionospheric Disturbances}

The GBR (Rugby)-Malta propagation path may support more than one mode of propagation by night and it has been put forward that only one mode is present during the daytime [Burgess 1963]. It is possible to test this hypothesis by observing the effect of an SID simultaneously on this path and on a path of much longer range. On 12th July 1961, a class $3+$ flare occurred at 10.20 U'T. Both the GBR-Malta and the NBA (Panama)-Rome propagation paths, monitored at the time, exhibited the effect of the flare. On the basis of one mode of propagation over the GBR-Malta path, the effect of

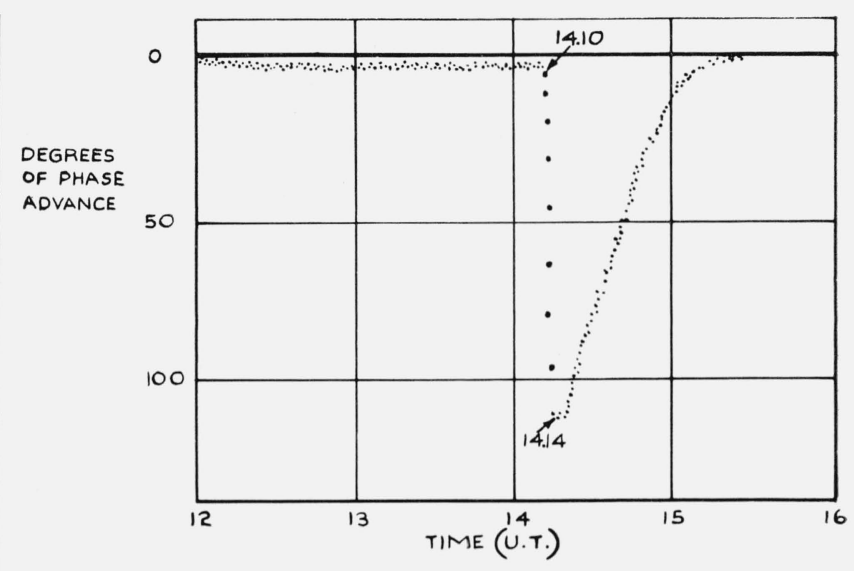

Figure 1. Sudden phase anomoly observed NBA-Malta pathe 27 A pril 1962.

the flare on both paths mentioned above are consistent.

The solar flare of class 2 on April 27, 1962, is of interest since information on the X-ray spectrum $\left(3-12^{\circ} \mathrm{A}\right)$ of this flare was collected on board the satellite ARIEL. The visual flare commenced at $13.50 \mathrm{UT}$, reached its maximum intensity at $14.13 \mathrm{U} T$, and ended at $14.40 \mathrm{U}$ T. The X-ray flux increased over most of this period but there was a sudden burst at $14.13( \pm 3)$ U'T, the intensity increasing by a factor of 40 at $6^{\circ} \mathrm{A}$ [Pounds 1962]. The phase of the VLF station NBA recorded at Rome, Italy (fig. 1), showed a sudden advance at $14.10 \mathrm{U} T$, with its maximum rate of change of phase at $14.13 \mathrm{UT}$. This shows remarkable correlation of increase in ionization in the lower $D$-region with the influx of X-rays in the $3-12^{\circ} \mathrm{A}$ band.

\subsection{Magnetic Storm Effects}

During two major magnetic storms of November 1960 and July 1961 the phase of the GBR signal received at Rome underwent diurnal variations which were some 5 to 10 times greater than normal. Figure 2 shows a plot of a typical day during the disturbed period and compares it with the normal variation to be expected at that time of year. During November 1960 the anomalous VLF phase records had already started some $20 \mathrm{hr}$ after the associated solar flare of 12 th November and continued for at least 9 days. There were a number of solar events during this period and hence the effects of any one event are not clear. 


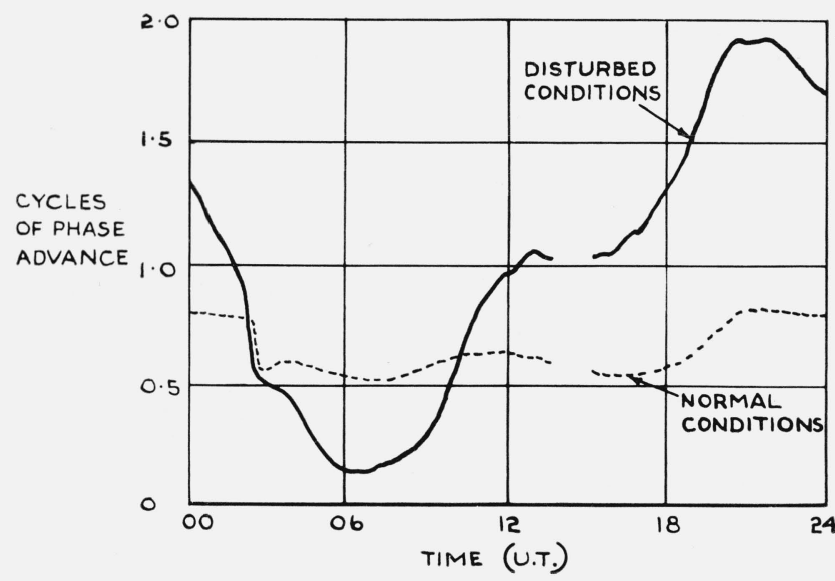

Figure 2. Diurnal phase variation of $G B R(16 \mathrm{kc} / \mathrm{s})$ at Rome under disturbed conditions.

During the July 1961 events, exactly the same form of phase variations were observed as in the previous November. From these results it seemed likely that the major VLF effects last for 2 days and then return to normal. A further example of this behavior occurred in September 1962, when once again the magnetic $K$ indices registered large values $(>K>7)$. In this case, progression of the phase trace from normal conditions to abnormal and back to normal occurs within 3 to 4 days. Other VLF propagation paths monitored during this period behaved in a reasonably normal fashion, indicating that the disturbance is confined to northern latitudes. The present summary paper cannot discuss the many facets of these observations which will be reported on in a more detailed paper.

\subsection{High Altitude Nuclear Bomb Effects}

Manmade disturbances in the upper atmosphere have similar characteristics to those which occur naturally.

During the period when the effects of the nuclear detonations would be expected to be observed
(July 1962, and mid-October to mid-November 1962), various VLF propagation paths were being monitored in connection with the R.A.E. long range navigation study. These paths included NPM (Hawaii)-Singapore, NPM-Farnborough, GBR-Singapore, GBRRome (Italy) and GBR-Malta. The initial full phase advancement due to the explosion could not be observed due to the long time constant of the receiving equipment. However, an advancement of phase, at, or beginning at the instant of detonation, was always observed and could be explained qualitatively either by direct radiation from the bomb or by neutron decay electrons, or electrons captured by the geomagnetic field and drifting eastward round the world.

In the case of NPM-Singapore path the effects of the American bombs lasted for approximately 8 to 12 days. This no doubt is due to the fission-produced ionization from debris still settling in the longitudes near the explosions. For these periods, the diurnal phase change of this path exhibited a trapezoidal pattern instead of the normal "double humped" diurnal phase variation. The depth of the diurnal phase trapezium was approximately $180 \mathrm{deg}$ of phase which is the value that would be expected from a single mode of propagation where the ionospheric boundary decreased in height by about 5 or $6 \mathrm{~km}$ from its nighttime to daytime level.

\section{References}

Bracewell, R. N., and K. G. Budden (1951), The ionospheric propagation of low- and very-low frequency radio waves over distances less than $100 \mathrm{~km}$, Proc. IEE. 98, Part III, 221.

Budden, K. G., and J. A. Ratcliffe (1939), Further investigations of very long waves reflected from the ionosphere, Proc. Roy. Soc. A1\%1, 188.

Burgess, B. (1963), Propagation of VLF waves, over distances between 1000 and $3000 \mathrm{~km}$, presented at the VLF Symposium, 12, 13, and 14 August, Boulder, Colo.

Chilton, C. J., D. D. Crombie, A. G. Jean (1962), Phase variations in VLF propagation, AGARDOGRAPH 74 (Pergamon Press, Oxford, London), in press.

Pounds, K. (1962), private communication.

(Paper 68D1-324) 